\title{
ВИРТУАЛЬНЫЙ МЕРЧЕНДАЙЗИНГ: СУЩНОСТЬ И ОСОБЕННОСТИ ПРИМЕНЕНИЯ В РАЗЛИЧНЫХ СФЕРАХ
}

\section{VIRTUAL MERCHANDISING: THE ESSENCE AND FEATURES OF ITS APPLICATION IN VARIOUS FIELDS}

\section{Yu. Selyavskii}

R. Kuksin

Summary: The theory and practice of merchandising has received a new impetus for development. The problem of using virtual merchandising in various fields of economic activity is reaching a new level. Since the 2000 s, scientific and practical interest in this topic has been growing. The author draws attention to the fact that this technology is changing the established approaches to marketing due to a number of arguments. The concept and economic essence of virtual merchandising has been clarified. The main functionality and possibilities of using VR / AR technologies for commodity science tasks are described. The author's positions in relation to virtual merchandising on the part of foreign researchers are analyzed. The systematization of researchers' opinions allows us to highlight their convergence in a number of fundamental points in the organization of virtual merchandising. By analyzing foreign experience, the author came to the conclusion that the number of possible methods of using new approaches to merchandising on the Internet is increasing in the world.

Keywords: merchandising, virtual reality, augmented reality, virtual merchandising, VR/AR technologies, XR technologies, e-tailing.
Селявский Юрий Валерьевич

К.э.н., Смоленский филиал ФГБОУ ВО «Российский экономический университет имени Г.В. Плеханова»

yurasel83@mail.ru

Куксин Роман Петрович

К.э.н., Смоленский филиал ФГБОУ ВО «Финансовый университет при Правительстве Российской Федерации»

romeon888@yandex.ru

Аннотация: Теория и практика мерчендайзинга получила новый импульс для развития. Проблема применения виртуального мерчендайзинга в различных сферах экономической деятельности выходит на новый уровень. Начиная с 2000-хгодов, растет научный и практический интерес к данной проблематике. Автор обращает внимание на тот факт, что данная технология меняет устоявшиеся подходы к маркетингу в силу целого ряда аргументов. Уточнено понятие и экономическая сущность виртуального мерчендайзинга. Описан основной функционал и возможности применения средств VR/ARтехнологий для задач товароведения. Проанализированы авторские позиции в отношении виртуального мерчендайзинга со стороны зарубежных исследователей. Систематизация мнений исследователей позволяет выделить их сближение по целому ряду принципиальных моментов в организации виртуального мерчендайзинга. Посредством анализа зарубежного опыта автор пришел к выводу о том, что в мире приумножается число возможных приемов использования новых подходов к мерчендайзингу в интернете.

Ключевые слова: мерчендайзинг, виртуальная реальность, дополненная реальность, виртуальный мерчендайзинг, VR/AR-технологии, XR-технологии, e-tailing.

в бизнесе. Следует подчеркнуть, что цикл современных инноваций очень сильно ускорился. Конкурентоспособность зависит от новых качеств, определяемых внедрением новых технологий и сетевых ресурсов развития. Развитие цифровых экосистем одновременно усиливает позиции бизнеса и ослабляет позиции регуляторов экономики, потому что возникает неопределенность в применении торговых правил из-за инноваций. На основе таких бизнес-моделей происходит децентрализация обмена товаров и услуг, а также развитие механизмов рыночного самоуправления. Следует исходить из того, что рост экономического оборота в цифровой экономике способен уничтожить традиционные бизнес-модели (оф-лайн-модели).

Начавшаяся эпидемия коронавируса (COVID-19) в 2020 г. повлияла на оптовую и розничную по всему миру: риск заражения инфекцией изменил структуру потребления и определил новые технологические вызовы в торговле. Эпидемия изменила привычки, досуг и образ жизни и работы потребителей: выросло количество онлайн-покупок, бесконтактных доставок товаров. Закры- 
тие границ определило логистические сбои и нехватку определенных групп товаров. Сами по себе торговые оф-лайн площадки рассматриваются в качестве идеальной среды для передачи коронавируса, так как большое количество покупателей прикасается к товарам, и перекладывает их. Конкуренция при пандемии COVID-19 изменила формы: меняются подходы к маркетингу, рекламе и электронной коммерции.

На современном этапе развития торговли активно применяются технологии виртуальной и дополненной реальности. Цифровизация торговли привела к появлению новых ранее не изученных подходов к организации бизнес-процессов. Новым трендом цифровизации торговли является развитие теории и практики виртуального мерчендайзинга, внимание к которому среди ученых в последнее время недостаточное. При этом практики высказывают мнение о необходимости разработки научных основ виртуального мерчендайзинга [3]. В то же время, анализ успешных практик виртуального мерчендайзинга показывает, что применение технологий этой группы позволяет бизнесу добиться неплохих результатов в контексте конкурентоспособности и эффективности. Необходимо подчеркнуть, виртуальный мерчендайзинг формирует экосистему организации, расширяет рыночное пространство. За счет технологий VR/AR возможности виртуального мерчендайзинга возрастают за счет интеграции с другими цифровыми инструментами (индустрия 4.0, экономика совместного пользования, социальные сети и цифровая торговля).

Обращение к инновационному опыту применения VR/AR-технологий привлекает внимание исследователей и требует внимательного изучения сущности и особенностей практики применения в различных сферах цифровой экономики. Для этого необходимо понимать специфику конкретного сектора экономики, в котором работает компания, желающая внедрить виртуальный мерчендайзинг. Обобщение мнений экспертов по внедрению показывает, что проблемной точкой внедрения виртуального мерчендайзинга как других технологий цифровой экономики является взаимодействие заказчика с интегратором, так как при этом приходиться терять контроль и делиться частью полномочий [2]. Вследствие чего нарушается экосистема предприятия, потому что нередко такие подходы противоречат корпоративной этике компаний.

Соответственно социальная актуальность заявленной проблематики выражается в том, что пора развивать концептуальные научные подходы к виртуальному мерчендайзингу (теория, социально-культурные связи и управление ими). Прикладной смысл данной концепции заключается в том, что правильно организованная данная деятельность способна влиять на чувства и настроения целевых аудиторий: вызывает чувства благодарно- сти и лояльности у клиентов и комфортные ощущения от покупки. Если этот эффект присутствует в деятельности торговых предприятий, то цели социальной эффективности достигнуты, так как понимание дизайна мышления потребителей в условиях цифровизации и пандемии COVID-19 является конкурентным преимуществом [9].

В рамках данной публикации основной целью статьи является анализ современных тенденций применения виртуального мерчендайзинга в различных отраслях экономики. Для этого ставились и решались следующие задачи исследования:

1. раскрытие экономической сущности виртуального мерчендайзинга;

2. анализ практического опыта виртуального мерчендайзинга.

\section{Методика}

Обращение к научной литературе позволяет выделить ограниченное количество научных исследований по проблематике виртуального мерчендайзинга. В основном научные исследования представлены учеными из Российской Федерации [1; 10], США [5], Евросоюз [4; 6], Китая [8; 12], Индии [13], Пакистана [9], Южной Кореи [7]. Не отстают от ученых и специалисты в области виртуального мерчендайзинга, публикации которых имеют не последнее значение для понимания научных основ виртуального мерчендайзинга [6].

Ключевые слова. Термин «виртуальный мерчендайзинг» прошел определенную эволюцию, что подтверждается богатым семантическим полем данного термина, представленного такими понятиями как «товароведение», «мерчендайзинг», «выкладка товаров», «снабжение товаров рекламными материалами», «визуальный мерчендайзинг в интернете», «электронный мерчендайзинг», «кросс-мерчендайзинг», «Е-мерчендайзинг», «интернет-мерчендайзинг», «он-лайн-мерчендайзинг, «виртуальный мерчендайзинг»,и др. Примечательно, что в появление каждого из перечисленных синонимов понятия «мерчендайзинг» детерминировано изменениями в формах, методах и технологиях, внедряемых в торговлю в России и зарубежных странах.

Кроме того, важнейшее значение имеет определение критериев для определения эффективности виртуального мерчендайзинга. К таковым на современном этапе следует отнести критерий рационального выбора за разумное время, который делает покупатель поверхностно знакомый с предметной областью.

\section{Основное изложение материала}

Термин «виртуальный мерчендайзинг» чаще всего понимается как феномен с разными трактовками. Боль- 
шое распространение получила точка зрения, согласно которой виртуальный мерчендайзинг является видом товароведения, который опирается на привычки и ассоциации клиентов, умелое манипулирование которыми повышает продажи товаров и услуг в несколько раз [3]. Следует признать, такое упрощенное понимание не раскрывает особенностей виртуального мерчендайзинга. Развитие интернет-магазинов обусловило интерес к виртуальному мерчендайзингу, где применяются принципиально другие инструменты привлечения внимания клиентов, учитывающие природу электронной торговли. Технологии дополненной и виртуальной реальности позволяют идентифицировать, вспомнить клиента, совершенные им покупки, интересы и предпочтения, способы оплаты и доставки. Данная информация особенно востребована для организации повторных продаж, сегментирования клиентов по ценовой категории и обратной связи с клиентами. Такой подход закономерно сближает клиентов с виртуальными продавцами.

Следует признать, наиболее полно раскрывает экономическую сущность виртуального мерчендайзинга российский коллектив исследователей Д. Ралык, Н. Иванова и Т. Горгодзе (2019). Исследователи определяют термин «виртуальный мерчендайзинг» как маркетинговый инструмент в сфере электронной розничной торговле, применение которого обеспечивает рациональную организацию информационного торгового пространства, когнитивных и временных ресурсов покупателей с учетом знаний психологии потребителя [11]. Авторы обращают внимание на тот факт, что с появлением виртуального мерчендайзинга для ритейлеров теряют значение физическая структура магазина и время его эксплуатации, и большее значение приобретает организация электронных торговых площадей.

Установлено, что изображение продукта и текст на дисплее стимулирует восприятие потребителей [8], а реклама 3D усиливает намерения совершить покупку [12], дает базовые знания, облегчает принятие решений о покупках [13]. Установлено, что обеспечение привлекательности веб-сайтов средствами VA/AR позволяет провоцировать у потребителей импульсные и незапланированные покупки, определяя тем самым дополнительный потенциал продаж [9].

Научный интерес представляют результаты прикладных исследований из Южной Кореи. Профессор в области маркетинга JihyePark (Hankuk, SouthKorea, 2005) в соавторстве c L. Khakimdjanova в совместной публикации уделяют внимание отраслевым практикам виртуального мерчендайзинга [7]. Посредством контент-анализа контента веб-сайтов магазинов одежды исследователи пришли к выводу о том, что проектирование методов виртуального мерчендайзинга позволяет создавать более привлекательную среду для совершения электрон- ных покупок, определяя большую конкурентоспособность Е-тейлеров по сравнению с ретейлерами.

К интересным выводам о роли виртуального мерчендайзинга приходит JoeBardi (2016) [4]. Применение в мерчендайзинге виртуальной и дополненной реальности вносит ряд корректив в торговую деятельность:

- разрушает традиционный визуальный маркетинг,

- обеспечивают обратную связь между клиентом и брендом,

- обеспечивают уникальный и интересный опыт покупки,

- каталогизация и генерация активности и поведения покупателей;

- обеспечение дополнительного быстро настраиваемого виртуального пространства, сезонных шаблонов, которое оперативно адаптируется к опыту, который ритейлер хочет спроектировать для клиента; снижает затраты (логистика, потеря образцов продукции, изготовление образцов) при демонстрации продукции.

Следует признать, что описанный функционал виртуального мерчендайзинга меняет отношение к нему, и позволяет рассматривать его как технологию менеджмента, обеспечивающую одновременно коммуникации и принятие решений, необходимых для компаний, а также оказывающую серьезное влияние на все функции менеджмента.

В своей работе «Руководство по мерчендайзингу» американский профессор lanaCastro (SanDiego, USA, 2019) обосновал возможный подход к визуальному мерчендайзингу [5]. Исследователь связывает визуальный мерчендайзинг с необходимостью управляемого взаимодействия продаваемых товаров и их потребителей на площадках розничной торговли: с момента доставки товара в магазин и до момента, когда клиент берет товар из полки в руки. По мнению исследователя, визуальный мерчендайзинг должен учитывать следующие организационные условия: нейромаркетинг, кросс-мерчендайзинг, всплывающие дисплеи (или "точка покупки" - POP displays). Само понимание ученым визуального мерчендайзинга как возможной тактики и стратегии товароведения наводит на мысль о большом многообразии его приемов.

В мире сегодня достаточно инновационных разработок в сфере виртуального мерчендайзинга. DanGuenther (2019) обращает внимание на моральное устаревание устоявшихся традиционных подходов к мерчендайзингу. Поэтому автор предлагает использовать мерчендайзинг виртуальной реальности как инновационный способ, основу которого составляют VR-гарнитуры с интегрированной функцией отслеживания глаз, применение которых дает возможность получать более целостные- 
данные при незначительной стоимости этого процесса. Автор обращает внимание на тот факт, что устоявшиеся традиционные подходы к мерчендайзингу более ограничены. Требуется соблюдение целого ряда процедур: проектирование дорогостоящей физической среды магазина, приглашение фокус-групп для тестирования полок и опрос фокус-групп. По качеству процедур более сильные позиции все-таки имеет виртуальный мерчендайзинг. Чтобы в этом убедиться, в компаниях Kellogg'S и Qualcommaвтор проанализировал практический опыт применения мобильных гарнитур виртуальной реальности с интегрированной функцией отслеживания глаз [6]. Был сделан вывод о том, что по целому ряду критериев виртуальный мерчендайзинг имеет ряд практических преимуществ по сравнению с традиционным мерчендайзингом:

1. улучшение опыта работы в фирменной среде функция отслеживания глаз позволяет получить дополнительную информацию о поведении потребителя (было выяснено, что помещение на нижнюю полку нового товара в комбинации с другими товарами на других полках, которым уделялось большее внимание, стимулирует дополнительные продажи нового продукта на 18\%);

2. увеличение набора данных для аналитики - технология отслеживания глаз позволяет не прерывать процесс покупок в виртуальном магазине;

3. расширяет охват тестирования географически рассредоточенных потребителей - для этого мобильные VR-гарнитуры позволяют потребителям тестировать продукт дома;

4. виртуальная реальность позволяет снизить затраты на тестирование планограмм (схем выкладки товаров), и делает данную процедуру более дешевой, чем в обычном офлайн магазине.

Таким образом, необходимо признать, что предложенный вариант технологии виртуального мерчендайзинга позволяет эффективно контролировать географически рассредоточенных групп потребителей, быстро анализировать данные и получать правильные системные маркетинговые выводы. Применение в технологии виртуального мерчендайзинга функции отслеживания глаз позволяет оптимально расставлять весь продуктовый ассортимент (виртуальные наборы продуктов).
Применение описанных технологий в виртуальном магазине позволит реализовывать эффективные сценарии тестирования потребителей в большом количестве географических регионов, тем самым формируя расширенную реальность.

\section{Выводы}

Подведение итогов статьи позволяет рассматривать виртуальный мерчендайзинг как прикладную технологию менеджмента, обеспечивающую коммуникации, принятие решений и цифровой маркетинг. В практическом аспекте виртуальный маркетинг позволяет рационально организовать информационное торговое пространство, сэкономить когнитивные и временные ресурсы покупателей. Использование средств VA/AR на веб-сайтах и мобильных приложениях потребителей импульсные и незапланированные покупки, определяя тем самым дополнительный потенциал продаж. Установлено, что умелое манипулирование привычками и ассоциациями клиентов повышает продажи товаров и услуг в несколько раз. Поэтому в виртуальном мерчендайзинге эксплуатируется способность VA/AR идентифицировать, вспомнить клиента, совершенные им покупки, интересы и предпочтения, способы оплаты и доставки. Основным критерием критерий эффективности виртуального мерчендайзинга выступает возможность рациональный выбора за разумное время, который делает покупатель поверхностно знакомый с предметной областью.

Анализ целого ряда работ (Д. Ралык, Jungmin, Y., Minjeong, Kim, Bingjia Shao, Jihye Park, Verma D.) показал, что виртуальный мерчендайзинг позволяет быстро адаптироваться к требованиям рынка, так как он вытесняет традиционные подходы к маркетингу. Исследования (Jihye Park, Joe Bardi, lana Castro) показывают, что основу виртуального мерчендайзинга составляет омниканальные цифровые устройства, нейромаркетинг и интернет. В исследованиях (Dan Guenther) отмечается, что посредством виртуального мерчендайзинга развиваются новые подходы к сегментации целевых аудиторий потребителей - появляется возможность мониторинга потребительского поведения и намерений географически рассредоточенных потребителей и формировать расширенную реальность.

\section{ЛИТЕРАТУРА}

1. Красюк И.А., Кхалаф К.З., Худик Д.Д. Инновации в сетевой торговле как инструмент формирования конкурентных преимуществ / И.А. Красюк, К.3. Кхалаф, Д.Д. Худик // Практический маркетинг. - 2017. - №2. - С.54-60.

2. 0 подходах к цифровой трансформации металлургических предприятий / (ред. 26.11.2019) // Эксперт on-line. - [Электронный ресурс]. - Режим доступа: https://expert.ru/2019/11/26/0-podhodah-k-tsifrovoj-transformatsii-metallurgicheskih-predpriyatij/ (дата обращения: 21.10.2020).

3. Стоит ли инвестировать в виртуальный мерчандайзинг? / Marketing.by. - [Электронный ресурс]. - Режим доступа: https://marketing.by (дата обращения: 22.10.2020). 
4. Bardi J. Top 4 reasons AR and VR are made for visual marketing / J. Bardi / Marxent 3D. - [Electronic resource]. - Access mode: https://www.marxentlabs.com (Date of Access: 24.10.2020).

5. Castro I. The ultimate guide to merchandising / I. Castro / Practical guide. - Boston: Repsly, 2019. - 9p.

6. Guenther D. Virtual reality merchandising (ed. 14.02.2019)/ D. Guenther / Research report. - [Electronic resource]. - Access mode: https://www.accenture.com/ us-en/insights/technology/virtual-reality-merchandising (Date of Access: 24.10.2020).

7. Park J. KhakimdjanovaL. Online visual merchandising practice of apparel e-merchants / J. Park, L.Khakimdjanova / Journal of Retailing and Consumer. - 2005. 12(5). - P.307-318.

8. Jungmin Y.; Minjeong Kim. The effects of online product presentation on consumer responses: A mental imagery perspective. J. Bus. Res. 2014.

9. Idrees S., Gill S., Vignali G. Technological Advancement in Fashion Online Retailing: A Comparative Study of Pakistan and UK Fashion E-Commerce / S. Idrees, [and other] // World Academy of Science, Engineering and Technology International Journal of Economics and Management Engineering. - 2020. - Vol14. - No4. P.313-328.

10. Morgan B. Five Trends Shaping The Future Of Customer Experience In 2020 (ed. 2019) / B. Morgan // Forbes. - [Electronic resource]. - Access mode: https://www. forbes.com/sites/blakemorgan/2019/12/17/5-predictions-for-customer-experience-in-2020/\#487b11cb2ec7 (date of access: 4.10.2020).

11. Ralyk D., Ivanova N., Gorgodze T. Virtual Merchandising As A Progressive Driver Of Retail In Digital Economic Conditions / D. Ralyk, N. Ivanova, T. Gorgodze // II International Scientific Conference GCPMED 2019 «Global Challenges and Prospects of the Modern Economic Development». - EpSBS / Volume 79 - GCPMED 2019. - P.766-772.

12. Rong Ma, Bingjia Shao, Jiaqi Chen and Dan Dai The Impacts of Online Clothes Short Video Display on Consumers' Perceived Quality / Ma Rong, [and other] // Information. - 2020. - №11(2). - 87 - [Electronic resource]. - Access mode: https://doi.org/10.3390/info11020087 (Date of Access: 24.10.2020).

13. Verma D., Jakhar R., Pal Singh Rathore A., Kumar D.Prioritization of Dimensions of Visual Merchandising for Apparel Retailers using Fuzzy AHP / D. Verma [and other] // Benchmarking An International Journal. - Vol. 27. - No.10. - pp.2759-2784.

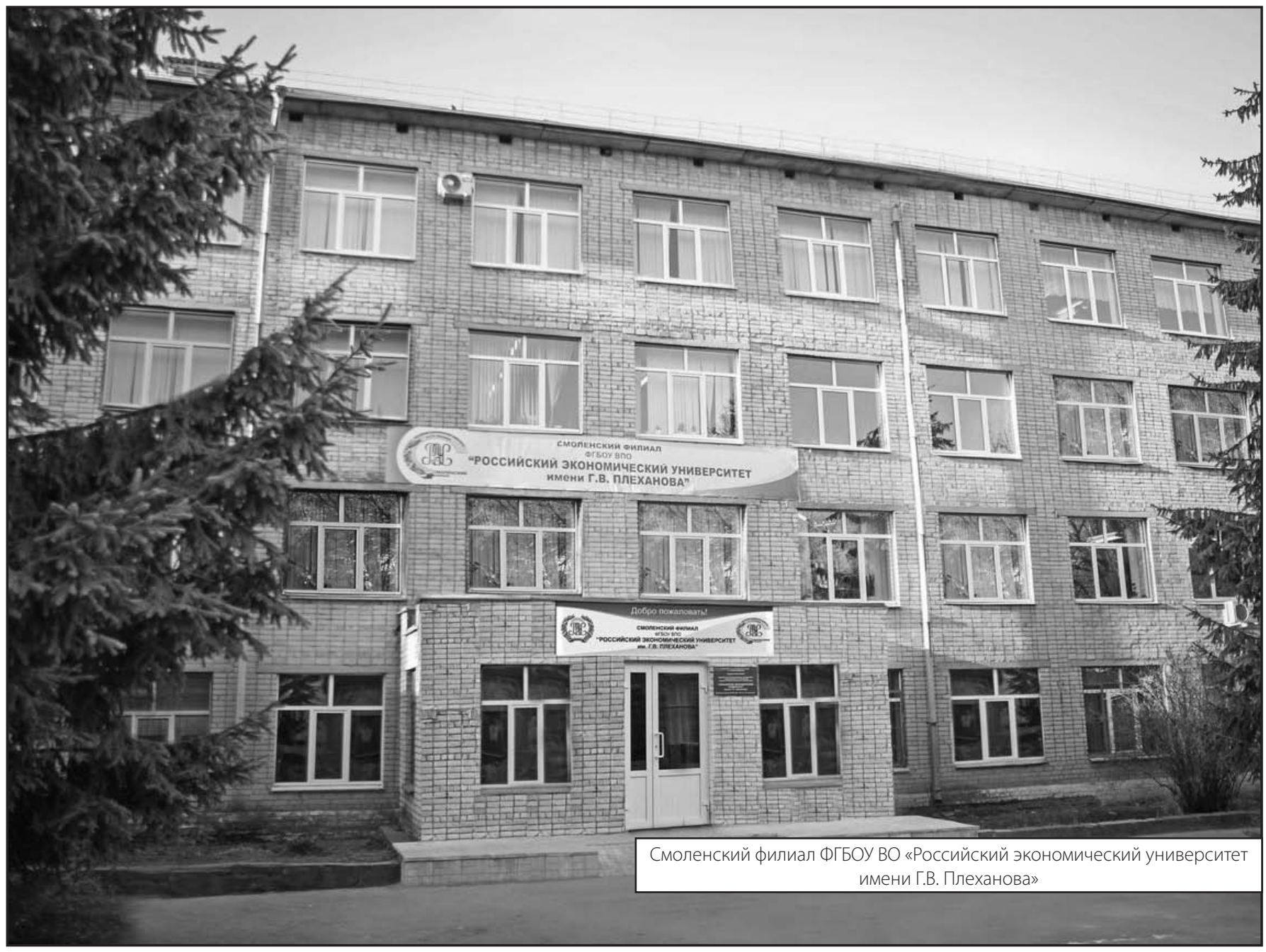

